\title{
Thermal activation and ATP dependence of the cytoskeleton remodeling dynamics
}

\author{
R. Sunyer, ${ }^{1,2}$ F. Ritort, ${ }^{3}$ R. Farré, ${ }^{1,4}$ and D. Navajas ${ }^{1,2, *}$ \\ ${ }^{1}$ Unitat de Biofísica i Bioenginyeria, Universitat de Barcelona and CIBER-Enfermedades Respiratorias, 08036 Barcelona, Spain \\ ${ }^{2}$ Institut de Bioenginyeria de Catalunya, 08028 Barcelona, Spain \\ ${ }^{3}$ Departament de Física Fonamental, Facultat de Física, Universitat de Barcelona and CIBER-BBN Networking Centre on Bioengineering, \\ Biomaterials, and Nanomedicine, 08028 Barcelona, Spain \\ ${ }^{4}$ Institut d'Investigacions Biomèdiques August Pi i Sunyer, 08036 Barcelona, Spain
}

(Received 21 November 2008; revised manuscript received 12 February 2009; published 27 May 2009)

\begin{abstract}
The cytoskeleton (CSK) is a nonequilibrium polymer network that uses hydrolyzable sources of free energy such as adenosine triphosphate (ATP) to remodel its internal structure. As in inert nonequilibrium soft materials, CSK remodeling has been associated with structural rearrangements driven by energy-activated processes. We carry out particle tracking and traction microscopy measurements of alveolar epithelial cells at various temperatures and ATP concentrations. We provide the first experimental evidence that the remodeling dynamics of the CSK is driven by structural rearrangements over free-energy barriers induced by thermally activated forces mediated by ATP. The measured activation energy of these forces is $\sim 40 k_{B} T_{r}$ ( $k_{B}$ being the Boltzmann constant and $T_{r}$ being the room temperature). Our experiments provide clues to understand the analogy between the dynamics of the living CSK and that of inert nonequilibrium soft materials.
\end{abstract}

DOI: 10.1103/PhysRevE.79.051920

PACS number(s): 87.16.Ka, 87.16.Ln, 87.17.-d

\section{INTRODUCTION}

The cell, an intricate biochemical factory, uses energetic supplies to carry out a myriad of specific cellular functions. Some of these functions such as locomotion and contraction involve generation of mechanical forces and structural rearrangements of subcellular structures $[1,2]$. The cytoskeleton (CSK) is responsible for force generation and for mechanical integrity of the cellular space. This complex structure is a nonequilibrium system in a state of steady remodeling sustained by the continuous expenditure of hydrolyzable sources of free energy such as adenosine triphosphate (ATP). The remodeling dynamics of the CSK has been compared with that of inert nonequilibrium soft materials such as foams, pastes, and slurries [3]. These materials are characterized by a marked dissipative viscoelastic response that requires a rearrangement of the relative positions of the molecules forming the structure of the material [4]. In the CSK, it has been suggested that these rearrangements are driven by shortranged forces that frustrate the large scale order and generate a strongly disordered and heterogeneous structure of entangled polymers [5]. The dynamics of these forces on the CSK reorganization has been interpreted in terms of a rugged free-energy landscape with structurally disordered states with random energies activated by a nonthermal effective temperature $[5,6]$. However, the phenomenological nature of this free-energy landscape has limited the application of this concept in cell biology, and its adequacy is a subject of intense controversy $[3,5,7-10]$.

Potential mechanisms that can generate random freeenergy barriers [6] include the potential-energy landscape set up by cytoskeletal motor proteins $[11,12]$ and polymerization of cytoskeletal filaments [13]. All these processes entail intramolecular and intermolecular weak interactions that are

\footnotetext{
*dnavajas@ub.edu
}

thermally activated and use ATP as a chemical source of free energy. The rate constants of thermally activated processes depend exponentially on temperature $(T)$ following the Arrhenius law $\propto \exp \left(-E_{0} / k_{B} T\right)$, where $E_{0}$ is the activation energy and $k_{B}$ is the Boltzmann constant. Under such conditions, we expect the remodeling dynamics of the CSK to be governed by free-energy barriers mediated by ATPdependent thermally activated force generators. Are temperature and intracellular ATP concentration the key variables that characterize the remodeling dynamics of the CSK? Does Arrhenius law describe the temperature dependence of cytoskeleton remodeling? Answering these questions is an important step toward a better comprehension of cellular mechanics.

The aim of this work is to ascertain whether the nonequilibrium remodeling dynamics of the CSK is governed by free-energy barriers from thermally activated forcegenerating processes. We probed structural rearrangements and forces of the CSK at various temperatures and intracellular ATP concentrations by means of two approaches: first, by monitoring spatial fluctuations of microbeads attached to the cell surface with particle tracking microscopy (PTM), and second, by assessing cytoskeletal stress fluctuations transmitted to the cell substrate by traction microscopy (TM). Our data show that CSK remodeling dynamics is thermally activated through ATP-dependent free-energy barriers.

\section{MATERIALS AND METHODS}

\section{A. Cell culture}

Human alveolar epithelial cells (A549) (cell line CCL185 ATCC, Manassas, VA) were cultured in RPMI 1640 medium supplemented with $1 \mathrm{mM} \mathrm{L-glutamine,} 100 \mathrm{U} / \mathrm{ml}$ penicillin, $100 \mathrm{mg} / \mathrm{ml}$ streptomycin and $2 \mu \mathrm{g} / \mathrm{ml}$ amphotericin B (all from GIBCO, Gaithersburg, MD), 10\% inactivated fetal calf serum (Biological Industries, Kibbutz Beit Haemek, Is- 
rael), and buffered with HEPES (Sigma, St. Louis, MO). One day before experiments cells were harvested with a brief exposure to trypsin ethylenediaminetetraacetic acid (Sigma) and plated $\left(900\right.$ cells $/ \mathrm{mm}^{2}$ ) on collagen-coated wells (for PTM measurements) or polyacrylamide gels (for TM measurements).

\section{B. CSK fluctuations probed by particle tracking microscopy}

CSK fluctuations were probed by tracking the movement of microbeads (4.5 $\mu \mathrm{m}$ diameter) attached to the apical surface of the cells (A549 cell size is $\sim 50 \mu \mathrm{m}$ ) [Figs. 1(a) and 1(b)]. The microbeads were previously coated with ArginineGlycine-Aspartic acid (RGD) containing peptide (Peptide 2000, Integra life Sciences, San Diego, CA) to link the probe to the actin CSK through integrin membrane receptors $[7,14]$. The time for the beads to attach to the cytoskeleton is in the order of tens of seconds [32]. Therefore, cells were incubated for $20 \mathrm{~min}$ after bead addition in order to maintain a firm and stable bead-actin coupling during measurements $[3,7,14]$. The mean-square displacement (MSD) of RGD coated microbeads is sensitive to a wide variety of CSK manipulations including actin polymerization/depolymerization, actomyiosin activation/relaxation, CSK stretching, temperature changes, and ATP depletion $[7,14,15]$, indicating that spatial fluctuations of microbeads linked to integrin membrane receptors reflect intrinsic CSK dynamics. An inverted microscope at $40 \times$ magnification (TE-2000E, Nikon, Japan) with a charge coupled device (CCD) camera (Orca, Hamamatsu, Japan) was used to record the spontaneous microbead movement for 200-400 s at a sampling frequency of $5 \mathrm{~Hz}$. Custom-made software (LABVIEW, National Instruments, U.S.) was implemented to track the position of the bead centroid $[r(t)]$ at subpixel resolution $[\sim 4 \mathrm{~nm}(\mathrm{rms})]$ by computing the microbead centroid with an intensityweighted average algorithm [16]. Data were corrected for the drift of the stage of the microscope, which was computed as the average change in the position of all microbeads within the field of view [7]. Instrumental noise was estimated by measuring MSD of beads glued to the substrate. However, $E_{0}$ estimated before and after subtracting the noise level from MSD data, varied $<0.1 \%$. To cool or heat the sample we used a microincubator system (HCMIS MicroIncubator System, ALA Science, Westbury, NY) closed-loop controlled. Sample temperature was measured with a negative temperature coefficient thermistor (332 Temperature Controller, Lakeshore, Westerville, $\mathrm{OH})$. Measurements were taken in $n=6$ wells $(\sim 20 \mathrm{microbead} /$ well $)$ per temperature and ATP concentration.

\section{Microbead motion analysis}

The trajectory of each microbead was characterized by computing the MSD defined as

$$
\left\langle\Delta r^{2}(\Delta t)\right\rangle=\left\langle[r(t+\Delta t)-r(t)]^{2}\right\rangle,
$$

where $\Delta t$ is the time lag and $t$ is the elapsed time. The mean of the logarithmic-transformed data was computed for all microbeads in the well. The MSD was fitted with

$$
\left\langle\Delta r^{2}(\Delta t)\right\rangle=\eta+\Gamma \Delta t^{\alpha}
$$

where $\alpha$ is the superdiffusion exponent, $\Gamma$ is a scale factor, and $\eta$ accounts for the subdiffusive contribution at short time scales and the instrumental noise [17]. The superdiffusive regime shows a slight decrease in the slope at large time scales. Accordingly, we fitted the above equation to MSD data before the appearance of the downturn in the slope of the MSD curve.

\section{Force fluctuation probed by traction microscopy}

We used TM to measure the stress fluctuations exerted by the cell on the substrate at different temperatures. We platted A549 cells on an elastic polyacrylamide gel with embedded refractive beads of $4.5 \mu \mathrm{m}$ in diameter. Stress fluctuations exerted by cells were transmitted to the substrate causing gel deformations. These deformations were measured by tracking the position of the embedded microbeads at $20 \times$ magnification with the same hardware and software used to track the position of CSK bound microbeads [accuracy $\sim 4.3 \mathrm{~nm}$ (rms)]. Traction forces exerted by the cells were computed using the force-deformation relationship of the polyacrylamide gel. Measurements were taken in $n=6$ wells per temperature $(\sim 30$ microbeads/well $)$.

Preparation of thin collagen-coated polyacrylamide gels for TM was carried out as described previously $[18,33]$. Briefly, we used $4.5 \mu \mathrm{m}$ refractive microbeads mixed with $2 \%$ acrylamide and $0.3 \%$ bis-acrylamide solution (BioRad, Richmond, CA). The solution polymerized with the addition of $10 \%$ of ammonium persulfate (Sigma, St Louis, MO) and subsequently tetramethylethylenediamine (BioRad). A fixed volume of $10 \mu \mathrm{l}$ of the resulting polyacrylamide solution was immediately placed onto a glass coverslip. Then, a 12 $\mathrm{mm}$ in diameter coverslip was placed on top of the polyacrylamide solution. To collapse the microbeads beneath gel surface we centrifuged the acrylamide-microbead solution during gelification at $300 \mathrm{rpm}$ for $10 \mathrm{~min}$. After $45 \mathrm{~min}$, the solution was completely polymerized and the $12 \mathrm{~mm}$ coverslip was carefully peeled off. Type I collagen $(200 \mathrm{mg} / \mathrm{ml})$ was added to the gel surface for $2 \mathrm{~h}$ to coat it.

\section{E. Force-deformation relationship of the polyacrylamide gel}

To compute the cytoskeletal stress fluctuations exerted on the soft polyacrylamide gel, we measured the stiffness $(k)$ that relates cell forces $(f)$ to gel displacements $(l)$ as $f=k l$. We measured $k$ by trapping polystyrene microbeads $(3 \mu \mathrm{m}$ in diameter) located below the gel surface with optical tweezers. After trapping the beads, we moved the optical trap at a constant velocity of $0.14 \mu \mathrm{m} / \mathrm{s}$ while monitoring the position and the lateral force applied to the microbead. We found a linear elastic behavior over a range $( \pm 550 \mathrm{~nm})$ covering the displacements and forces measured in stress fluctuation experiments (Fig. 2). Taking into account the stiffness of the optical trap $\left(k_{\text {trap }}=54 \mathrm{pN} / \mu \mathrm{m}\right)$, the actual stiffness of the gel is given by 


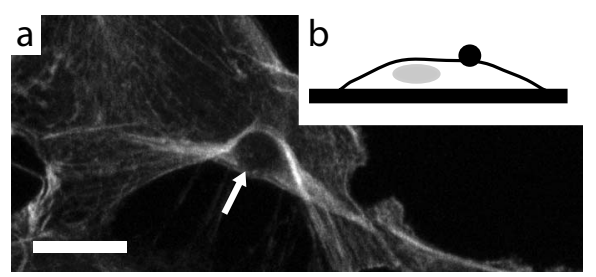

FIG. 1. (a) Immunoflorescence image (Leica confocal microscope, $\times 63$ objective, $z$ projection) of alveolar epithelial cells (A549) stained to show the actin filaments. RGD coated refractive microbeads (arrow) bind to the actin CSK via focal adhesion complexes (integrins). Scale bar is $10 \mu \mathrm{m}$. (b) Cytoskeletal structural rearrangements probed by PTM of refractive microbeads attached to the apical cell surface.

$$
k=\frac{1}{k_{\text {slope }}^{-1}+k_{\text {trap }}^{-1}},
$$

where $k_{\text {slope }}$ is the slope of the $f-l$ curve. We obtained $k=208 \pm 61 \mathrm{pN} / \mu \mathrm{m}$ (mean $\pm \mathrm{SD}, n=3$ gel samples, and 4 microbeads per sample).

\section{F. Stress fluctuation analysis}

The stress fluctuations were characterized by computing the variance of the cellular force $(S)$ as

$$
S(\Delta t)=\left\langle[f(t+\Delta t)-f(t)]^{2}\right\rangle,
$$

where $f(t)$ is cellular force at time $t$ and $\Delta t$ is the time lag. The mean of the logarithmic-transformed data was computed for all microbeads in the well.

\section{G. Measurement of the gel stiffness at different temperatures}

Since our optical tweezers setup does not allow us to change temperature, we used atomic force microscopy to measure the Young's modulus of the gel at different temperatures. First, a V-shaped cantilever (nominal spring constant $k_{A F M}=0.01 \mathrm{~N} / \mathrm{m}$, Veeco, Santa Barbara, CA) was calibrated by the thermal fluctuation method $[19,20]$. The relationship between the photodiode signal and the cantilever deflection was calibrated before gel measurements as the slope of the force displacement curve obtained at a bare region of the coverslip. Force-displacement curves $(1 \mu \mathrm{m}$ indentation, 1 $\mathrm{Hz}$ ) were obtained over five separated gel regions. The Young's modulus of the gel was estimated by fitting indentation data with the Hertz pyramidal model [21]. We performed this protocol at 24 and $37{ }^{\circ} \mathrm{C}(n=5$ samples per temperature). The Young's modulus obtained was $194 \pm 28$ and $220 \pm 10 \mathrm{~Pa}($ mean $\pm \mathrm{SE})$ at 24 and $37{ }^{\circ} \mathrm{C}$, respectively. The confidence interval overlap of these two values indicates that gel stiffness can be considered invariant over the temperature range of our TM experiments.

\section{H. ATP depletion}

ATP depletion was carried out by incubating cells at $37{ }^{\circ} \mathrm{C}$ with $2-10 \mathrm{mM} \mathrm{NaN}_{3}$ and $10-50 \mathrm{mM}$ of deoxyglucose (Sigma) for $1 \mathrm{~h}$. Intracellular ATP levels were determined by

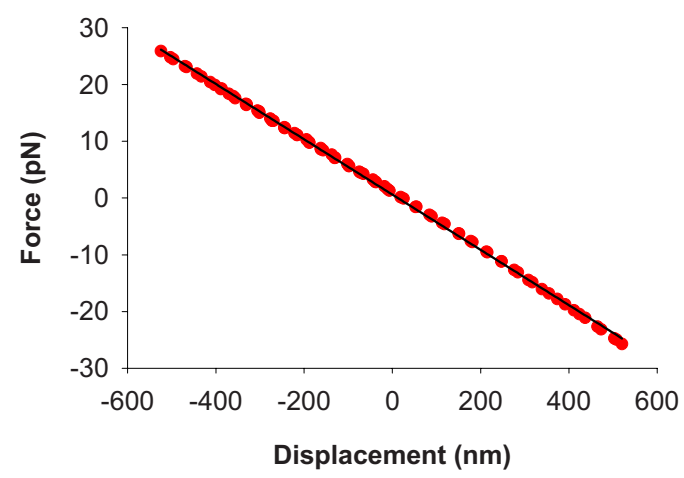

FIG. 2. (Color online) Representative force-displacement curve obtained by trapping a polystyrene microbead embedded in a polyacrylamide gel. Dark gray (red) dots are experimental data and black line is a linear fit $\left(r^{2}=0.99\right)$.

a bioluminescent somatic cell assay (Sigma). The ATP content of every measured well was determined in all ATP depletion experiments. To rule out the possibility that temperature slows down ATP supplying processes, we measured the ATP contents of cells at different temperatures. ATP concentration changed by only $1 \%$ as temperature varied from 37 to $13{ }^{\circ} \mathrm{C}$ ( $n=6$ wells per temperature). No significant differences were found among ATP levels at different temperatures [one-way analysis of variance (ANOVA), $P=0.461]$.

\section{RESULTS AND DISCUSSION}

Molecular scale structural rearrangements were assessed by tracking spatial fluctuation of microbeads attached to the apical surface of alveolar epithelial cells (A549) and linked to CSK via integrin receptors as described in Sec. II [Figs. 1(a) and 1(b)]. Each microbead undertook a random motion as the region of CSK to which it was attached reorganized. We characterized the CSK rearrangements at different temperatures $\left(13-37^{\circ} \mathrm{C}\right)$ by computing the $\operatorname{MSD}\left\{\left\langle\Delta r^{2}(\Delta t)\right\rangle\right.$ $\left.=\left\langle[r(t+\Delta t)-r(t)]^{2}\right\rangle\right\}$ of the microbead trajectory $r(t)$ as a function of time lag $(\Delta t)$ [Fig. 3(a)]. According to the value of the logarithmic slope $(\alpha)$, the MSD presents two distinct regimes: at short time scales it exhibits subdiffusion $(\alpha<1)$ associated with confined or caged motion of the microbeads, whereas at long time scales it shows superdiffusion $(\alpha>1)$ [9] associated with structural rearrangements through remodeling events of the CSK [7]. The transition from subdiffusion to superdiffusion cannot be attributed to the time scale of the integrin signaling cascade responsible for coupling the bead to the actin network since this time scale is in the order of tens of seconds [32], whereas the beads in our study were incubated on the cell surface for $20 \mathrm{~min}$ prior to MSD measurements. The superdiffusion regime shifts to longer times as temperature decreases. All data collapse into a master curve by appropriately rescaling the time of all MSD curves (taking the MSD at physiological temperature as the reference curve) [Fig. 3(b)]. For all temperatures, the superdiffusive regime of the MSD increases with time as a power law $\left[\left\langle\Delta r^{2}(\Delta t)\right\rangle \propto \Delta t^{\alpha}\right]$. The power-law exponent $(\alpha)$ takes a value of $1.58 \pm 0.06$ (mean $\pm \mathrm{SD}$ ) at $37^{\circ} \mathrm{C}$. A superdiffusive regime with a similar exponent at a comparable time scale has 


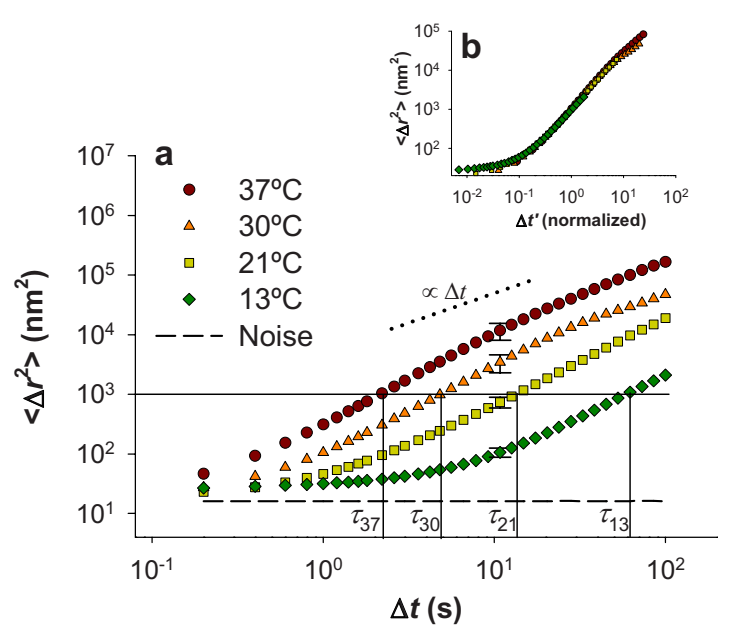

FIG. 3. (Color online) Dependence of cytoskeletal structural rearrangements on temperature. (a) Dependence on temperature of MSD of microbead fluctuations. The time scale of the superdiffusive regime at each temperature $T$ is characterized as the time $\left(\tau_{T}\right)$ required to achieve a given threshold of MSD (taken at $1000 \mathrm{~nm}^{2}$, solid black lines). The dashed line is the instrumental noise. (b) Data of panel a collapse into a master curve by appropriate rescaling of time by taking the data at $37^{\circ} \mathrm{C}$ as the reference curve.

been reported by tracking fluctuations of internalized microbeads [22] and endogenous lipid granules $[10,11]$ of different cell types at a single temperature. This indicates that MSD of microbeads attached to integrin membrane receptors can be taken as a readout of the intrinsic remodeling dynamics of the CSK matrix. The value of $\alpha$ remains almost constant over the temperature range explored, indicating that temperature does not significantly modify the superdiffusive regime of the CSK.

To assess the temperature dependence of the CSK remodeling dynamics, we characterize the time scale of the superdiffusion regime as the time $(\tau)$ required to achieve a given threshold of MSD [14] (which we take as $1000 \mathrm{~nm}^{2}$ ) [Fig. $3(\mathrm{a})]$. The precise value of this threshold is not relevant provided that it falls within the CSK superdiffusive regime. The remodeling rate $\left(\nu=\tau^{-1}\right)$ rises with temperature according to the Arrhenius law $\nu=A \exp \left(-E_{0} / k_{B} T\right), A$ being a prefactor that depends on the attempt frequency of remodeling events, the value of the MSD threshold used to extract $\nu$, and the extent of bead embedding into the cell (Fig. 4, triangles). A similar temperature dependence and $E_{0}$ value are obtained by taking different MSD thresholds within the superdiffusive regime. The Arrhenius behavior is a characteristic of thermally activated processes, e.g., chemical reactions or molecular transformations involving thermal transitions over free-energy barriers [23]. From the Arrhenius dependence of the CSK remodeling rate, we determine the value of the activation energy as $E_{0}=40 k_{B} T_{r}\left(r^{2}=0.99\right)$. A close value $\left(39 k_{B} T_{r}, r^{2}=0.98\right)$ is obtained by using an alternative method where we analyze the dynamic susceptibility associated with the motion of the microbeads [24]. Is it not $40 k_{B} T_{r}$ a too high free energy for thermal fluctuations be capable of inducing remodeling events? This apparently elevated effective energy could arise from complex processes involving multiple activated steps. Although thermal energy alone is

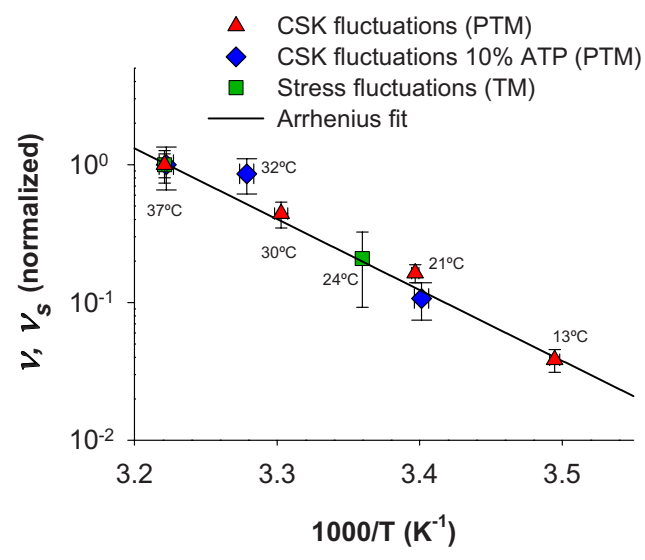

FIG. 4. (Color online) Arrhenius plot of the CSK remodeling rate $(\nu)$ at physiological ATP concentration $\left(c_{0}\right)$ (triangles) and at $10 \%$ ATP concentration (diamonds) and of the stress fluctuation rate $\left(\nu_{s}\right)$ at $c_{0}$ (squares). All measurements are normalized by the value at physiological temperature $\left(37^{\circ} \mathrm{C}\right)$. The line is the Arrhenius fit of $\nu$.

clearly insufficient to overcome $E_{0}$, the energy released by the ATP hydrolysis provides the nonthermal source of energy necessary to activate the remodeling events. CSK structural rearrangements at the molecular scale have been interpreted in terms of collective dynamics over free-energy barriers [3]. Our results provide evidence that CSK remodeling is driven by structural rearrangements over free-energy barriers. The relevant energy for these barriers has recently been a matter of speculation $[7,14]$. Here, we report for the first time a direct measurement of the energy scale governing CSK remodeling dynamics.

For each temperature, the remodeling rate computed from each individual bead $\left(\nu_{i}\right)$ shows some degree of heterogeneity that is compatible with a log-normal distribution extending over 2 orders of magnitude [Fig. 5(a) and [24]]. As each bead is attached to a different individual cell, the distribution of $\nu_{i}$ not only reflects the heterogeneity among beads but also among cells. The dispersion of $\nu_{i}$ could be explained by a distribution of the energy barriers among beads and cells that could induce, via Arrhenius law, a disparity of the relaxation times. To investigate this possibility, we compute the barrier $\left(E_{i}\right)$ corresponding to $\nu_{i}$ as $E_{i}=-k_{B} T \log \left(\nu_{i} / A\right)$, where we assume that the value of the prefactor $A$ is the same for all beads and cells. For each temperature the histogram of $E_{i}$ is equally distributed indicating that the energy distribution is temperature independent [Fig. 5(b)]. Accordingly, the distribution of free-energy barriers among beads and cells can be estimated by pooling the $E_{i}$ values from different temperatures and plotting them in a single histogram [Fig. 5(c)]. Could the degree of heterogeneity observed among beads and cells in our measurements have a dynamical origin? Many nonequilibrium glassy materials exhibit kinetic heterogeneity that has been attributed to thermally activated processes across randomly distributed free-energy barriers $[25,26]$. In addition, the dynamics of cytoskeleton was described by a broad distribution of free-energy barriers [3]. We found that the standard deviation of the resulting distribution is $0.4 k_{B} T_{r}$, indicating a narrow dispersion of the en- 


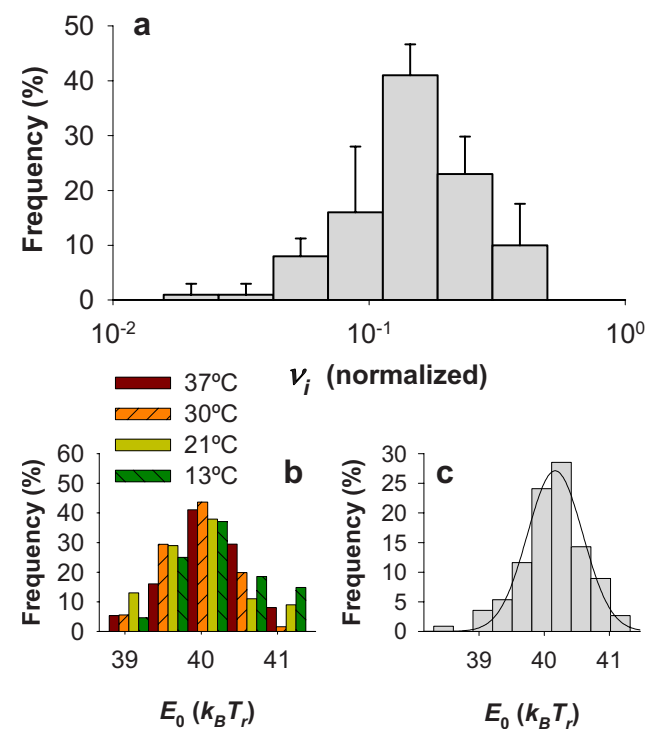

FIG. 5. (Color online) (a) Histogram of the CSK remodeling rate computed from each individual bead $\left(\nu_{i}\right)$ at $21{ }^{\circ} \mathrm{C}$ normalized by the value at physiological temperature $\left(37^{\circ} \mathrm{C}\right)$. (b) Histogram of free-energy barriers computed at different temperatures. (c) Histogram of free-energy barriers computed by pooling data at different temperatures. The solid line is a Gaussian fit centered at $40 k_{B} T_{r}$ with standard deviation of $0.4 k_{B} T_{r}$.

ergy barriers. This result suggests that $E_{0}$ does not vary much among beads and cells. Although we cannot exclude a kinetic origin, the heterogeneity observed in our experiments probably arises due to other factors such as a variable microbead diameter and a heterogeneous degree of embedding into the cellular membrane. Our results suggest that the notion of a broad distribution of energy barriers applied to cytoskeletal remodeling needs to be revisited.

To investigate whether structural rearrangements are induced by force generators we conducted TM experiments to measure CSK stress fluctuations. We platted the cells on a soft polyacrylamide gel ( $\sim 200 \mathrm{~Pa}$, see Sec. II) with embedded refractive microbeads. Cellular forces were quantified at 24 and $37{ }^{\circ} \mathrm{C}$ by tracking the displacement of the embedded microbeads placed below the gel surface [17] [Fig. 6(a)]. Force $(f)$ was computed from gel displacement $(l)$ as $f=k l$, where $k$ is the effective gel stiffness assessed by optical tweezers $(k=208 \pm 61 \mathrm{pN} / \mu \mathrm{m})$ (see Sec. II and Fig. 1). The variance of force fluctuations $(S)$ exhibits the same time dependence as the MSD probed with microbeads attached to the apical cell surface [Fig. 6(b)]. This parallel behavior lends further support to the adequacy of PTM measurements of microbeads linked to integrin membrane receptors for probing remodeling dynamics of the CSK. At $37{ }^{\circ} \mathrm{C}$ and $\Delta t>1 \mathrm{~s}, S$ increases with time as a power law with the same exponent $(\sim 1.5)$ as that exhibited by microbead superdiffusion [Fig. 3(a)]. Also consistent with the temperature dependence of structural fluctuations, the reduction in temperature to $24{ }^{\circ} \mathrm{C}$ shifts the power-law regime of stress fluctuations to longer times while maintaining the exponent constant. As in spatial fluctuations, we define the time scale of the stress fluctuations as the time $\tau_{s}$ required to achieve a given threshold of $S$ in the power-law regime $\left(10 \mathrm{pN}^{2}\right)$. The temperature

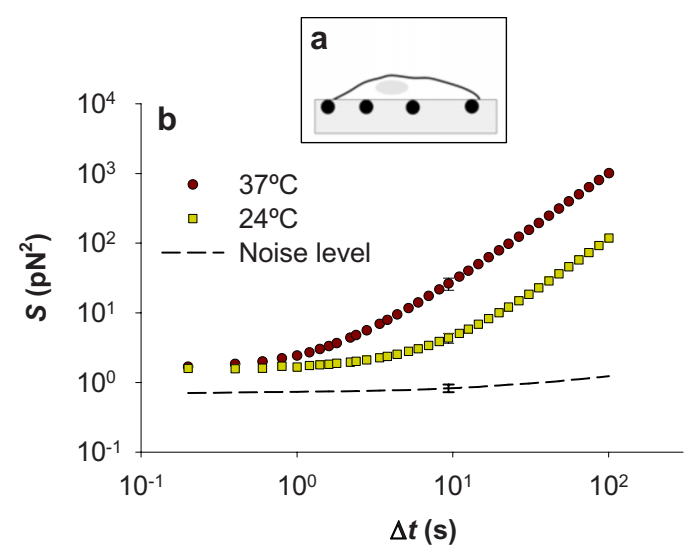

FIG. 6. (Color online) Dependence of cytoskeletal stress fluctuations on temperature. (a) Cytoskeletal stress fluctuations probed by TM in cells plated onto a soft elastic gel with embedded microbeads. Stress fluctuations are computed from microbead displacements measured by particle tracking. (b) Variance of force fluctuations $(S)$ at 24 and $37{ }^{\circ} \mathrm{C}$. Data are obtained from $n=6$ wells per temperature $(\sim 30$ microbeads/well). Error bars are $\mathrm{SD}$ at $\Delta t$ $=10 \mathrm{~s}$. The dashed line is the instrumental noise assessed from fluctuations of microbeads placed below the surface of the elastic gel without cells.

dependence of the stress fluctuation rate $\left(\nu_{s}=\tau_{s}^{-1}\right)$ is again consistent with the Arrhenius behavior observed in particle tracking experiments (Fig. 4 squares). This suggests that CSK stress fluctuations (as probed by TM) and structural rearrangements (as measured by PTM) originate from the same thermally activated force generators. It should be noted that the time window of our measurements is in the range of that of studies reporting activity of CSK molecular motors $[12,27]$. Taken together, our findings underscore that cytoskeletal remodeling dynamics is driven by force generators responsible for cell contraction and cytoskeletal internal tension, suggesting that the rearrangement of the cytoskeletal elements is closely connected to prestress generation.

How the remodeling dynamics depends on ATP concentration? ATP drives the cell into a nonequilibrium steady state where a fraction of the available free energy from hydrolysis is lost in the form of heat to the environment. This additional energy allows the CSK to overcome the energy barriers of $\sim 40 k_{B} T_{r}$ in observable time scales, thereby ATP concentration is expected to influence the remodeling kinetics of the CSK. To assess how ATP modifies the energetic balance inside the cell we carried out PTM measurements at different ATP concentrations $(c)$ by depleting intracellular ATP with different concentrations of sodium azide and deoxyglucose (see Sec. II). In a first series of experiments, we measured the temperature dependence at $10 \%$ ATP concentration. The temperature dependence of $\nu$ at $10 \%$ ATP is consistent with the Arrhenius law at physiological concentration $\left(c_{0}\right)$ (Fig. 4 , diamonds). In a second series of experiments, we assessed the ATP dependence of the remodeling rate at fixed temperatures [Fig. 7(a)]. In agreement with the kinetics of enzyme-catalyzed processes, $\nu$ decreases with ATP availability as the Michaelis-Menten equation [23] 

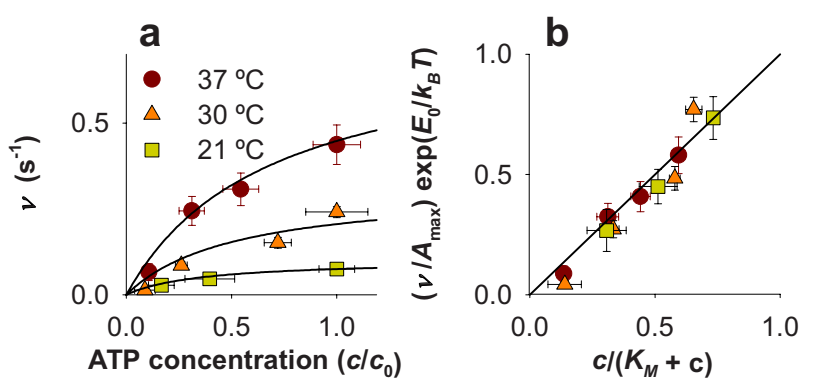

FIG. 7. (Color online) Dependence of cytoskeletal structural rearrangements on intracellular ATP concentration. (a) Remodeling rate $(\nu)$ versus intracellular ATP concentration $\left(c / c_{0}\right.$, with $c_{0}$ being physiological ATP concentration) at different temperatures $\left(21-37^{\circ} \mathrm{C}\right)$. The solid lines are fits of the Michaelis-Menten equation. (b) Remodeling rate in panel a normalized by the Arrhenius law and the Michaelis-Menten equation. The solid line is the identity.

$$
\nu(c, T)=\nu_{\max }(T) \frac{c}{K_{M}+c},
$$

where $K_{M}$ is the Michaelis-Menten constant and $\nu_{\max }(T)$ is the remodeling rate at $c \rightarrow \infty$ [solid lines in Fig. 7(a)]. The Michaelis-Menten kinetics assumes that the substrateenzyme reaction takes place in a homogeneous medium (i.e., well-mixed three-dimensional space and dilute conditions). Many biochemical or cellular processes deviate significantly from such conditions. This is particularly relevant in cell interior in which molecular crowding potentially frustrates the applicability of Michaelis-Menten. Therefore, the observation of Michaelis-Menten in our experiments indicates that the collective forces that induce cytoskeletal remodeling events can be described by simple enzyme kinetic laws. The maximum rate of the Michaelis-Menten fit rises with temperature according to the Arrhenius law $\left[\nu_{\max }(T)\right.$ $=A_{\text {max }} \exp \left(-E_{0} / k_{B} T\right)$, with $A_{\text {max }}$ being the Arrhenius prefactor corresponding to $\nu_{\text {max }}$ ]. A similar behavior is observed from dynamic susceptibility data [24]. From the fit of Eq. (1) to pooled $\nu$ data we obtain $K_{M} / c_{0}=0.7$ at $37{ }^{\circ} \mathrm{C}$, which corresponds to $K_{M} \sim 1.8 \mathrm{mM}$ given the physiological concentration of our cell type $\left(c_{0} \sim 2.7 \mathrm{mM}\right)[28] . K_{M} / c_{0}$ decreases to 0.4 at $21{ }^{\circ} \mathrm{C}$, indicating a weak temperature dependence of the Arrhenius prefactor. The dependence of the remodeling rate on temperature and ATP concentration causes data in Fig. 7(a) to collapse onto a master curve when $\nu$ is normalized by the Arrhenius law and the Michaelis-Menten equation [Fig. 7(b)]. Single molecule studies of motors proteins involved in transport processes (such as kinesin [29] or myo$\sin [30])$ have been observed to follow the Michaelis-Menten equation. The fact that the remodeling rate also follows the Michaelis-Menten kinetics suggests that molecular motors inside the cell are responsible for the remodeling dynamics of the CSK.

The superdiffusive behavior exhibited by CSK remodeling indicates persistent movement and mechanical work. In addition, intracellular ATP availability increases the remod- eling rate following the Michaelis-Menten equation. Although we cannot exclude other types of proteins, this behavior is characteristic of molecular motors, which use ATP to drive conformational changes in their globular domains that translate into movement through their coupling to cytoskeletal structures. Our stress fluctuation experiments suggest that remodeling is caused by force-generating motors coupled to cytoskeletal elements. This interpretation raises the question: are free-energy barriers modified by the CSK filaments under tension? Hypothetically, the force generated by molecular motors would increase CSK internal tension. An increase in this tension would modify the free energy of the barriers associated with remodeling events of cytoskeletal filaments. In this scenario, the free-energy barriers that determine the structural rearrangements of the CSK would originate from the competition between the force generated by the motors and the tension borne by the filaments. In agreement with this interpretation, earlier work on motility assays where in vitro actin filaments were moved by collective myosin motors reported energies of $(40-50) k_{B} T_{r}$ $[27,31]$, matching the activation energy values that we found. These observations give rise to a picture of the CSK where remodeling dynamics is governed by a ensemble of random free-energy barriers generated by the coupling between force generators and prestressed filaments.

\section{CONCLUSIONS}

We explored the physical laws that govern the temperature and the ATP dependencies of cytoskeleton remodeling. Nonequilibrium remodeling events, as probed by PTM and TM, are governed by thermally activated force generators following the Arrhenius law with an activation energy of $\sim 40 k_{B} T_{r}$. This remodeling kinetics increases with intracellular ATP availability and follows the Michaelis-Menten law. The Arrhenius behavior observed in cells is reminiscent of what has been observed in nonequilibrium systems where thermally activated processes lead to cooperative structural rearrangements. The Arrhenius and Michaelis-Menten laws bridge the gap between the views of cell biology and soft condensed-matter physics and provide a physical framework in which to unravel the controversial analogy between the dynamics of the living CSK and the behavior of inert nonequilibrium soft materials.

\section{ACKNOWLEDGMENTS}

We thank J. J. Fredberg and I. Pagonabarraga for critically reading the paper and $\mathrm{X}$. Trepat, $\mathrm{P}$. Roca-Cusachs, and $\mathrm{F}$. Rico for stimulating discussions. The technical assistance of M. Rodríguez, S. de Lorenzo, and the Laboratori Dr. Borrell are also acknowledged. This work was supported in part by Ministerio de Ciencia e Innovación Grants No. SAF200802991, No. NAN2004-09348, No. FIS2007-61433, and No. FIS-PI081908 and Generalitat de Catalunya Grant No. SGR05-00688. R.S. was supported by Departament d'Innovació i Empresa of the Catalan Government and the European Social Fund. 
[1] M. Chicurel, C. Chen, and D. Ingber, Curr. Opin. Cell Biol. 10, 232 (1998).

[2] P. Janmey, Physiol. Rev. 78, 763 (1998).

[3] B. Fabry, G. N. Maksym, J. P. Butler, M. Glogauer, D. Navajas, and J. J. Fredberg, Phys. Rev. Lett. 87, 148102 (2001).

[4] P. Sollich, F. Lequeux, P. Hebraud, and M. E. Cates, Phys. Rev. Lett. 78, 2020 (1997).

[5] B. Fabry and J. Fredberg, Respir. Physiol. Neurobiol. 137, 109 (2003).

[6] B. Fabry, G. N. Maksym, J. P. Butler, M. Glogauer, D. Navajas, N. A. Taback, E. J. Millet, and J. J. Fredberg, Phys. Rev. E 68, 041914 (2003).

[7] P. Bursac, G. Lenormand, B. Fabry, M. Oliver, D. Weitz, V. Viasnoff, J. Butler, and J. Fredberg, Nature Mater. 4, 557 (2005).

[8] M. Balland, N. Desprat, D. Icard, S. Fereol, A. Asnacios, J. Browaeys, S. Henon, and F. Gallet, Phys. Rev. E 74, 021911 (2006).

[9] B. Hoffman, G. Massiera, K. Van Citters, and J. Crocker, Proc. Natl. Acad. Sci. U.S.A. 103, 10259 (2006).

[10] K. Van Citters, B. Hoffman, G. Massiera, and J. Crocker, Biophys. J. 91, 3946 (2006).

[11] A. W. C. Lau, B. D. Hoffman, A. Davies, J. C. Crocker, and T. C. Lubensky, Phys. Rev. Lett. 91, 198101 (2003).

[12] D. Mizuno, C. Tardin, C. Schmidt, and F. MacKintosh, Science 315, 370 (2007).

[13] G. Danuser and C. Waterman-Storer, Annu. Rev. Biophys. Biomol. Struct. 35, 361 (2006).

[14] X. Trepat, L. Deng, S. S. An, D. Navajas, D. J. Tschumperlin, W. T. Gerthoffer, J. P. Butler, and J. J. Fredberg, Nature (London) 447, 592 (2007).

[15] P. Bursac, B. Fabry, X. Trepat, G. Lenormand, J. Butler, N. Wang, J. Fredberg, and S. An, Biochem. Biophys. Res. Commun. 355, 324 (2007).

[16] X. Trepat, M. Grabulosa, L. Buscemi, F. Rico, B. Fabry, J.
Fredberg, and R. Farre, Rev. Sci. Instrum. 74, 4012 (2003).

[17] C. Raupach, D. P. Zitterbart, C. T. Mierke, C. Metzner, F. A. Muller, and B. Fabry, Phys. Rev. E 76, 011918 (2007).

[18] R. Pelham and Y. Wang, Proc. Natl. Acad. Sci. U.S.A. 94, 13661 (1997).

[19] J. Hutter and J. Bechhoefer, Rev. Sci. Instrum. 64, 1868 (1993).

[20] H. Butt and M. Jaschke, Nanotechnology 6, 1 (1995).

[21] F. Rico, P. Roca-Cusachs, N. Gavara, R. Farre, M. Rotger, and D. Navajas, Phys. Rev. E 72, 021914 (2005).

[22] A. Caspi, R. Granek, and M. Elbaum, Phys. Rev. Lett. 85, 5655 (2000).

[23] J. Howard, Mechanics of Motor Proteins and the Cytoskeleton (Sinauer Associates, Massachusetts, 2001).

[24] See EPAPS Document No. E-PLEEE8-79-106905 for supplementary text. For more information on EPAPS, see http:// www.aip.org/pubservs/epaps.html

[25] M. Ediger, Annu. Rev. Phys. Chem. 51, 99 (2000).

[26] L. Cipelletti and L. Ramos, J. Phys.: Condens. Matter 17, R253 (2005).

[27] D. Winkelmann, L. Bourdieu, A. Ott, F. Kinose, and A. Libchaber, Biophys. J. 68, 2444 (1995).

[28] X. Lu, J. Errington, V. Chen, N. Curtin, A. Boddy, and D. Newell, Clin. Cancer Res. 6, 271 (2000).

[29] K. Visscher, M. Schnitzer, and S. Block, Nature (London) 400, 184 (1999).

[30] M. Nishikawa, H. Takagi, T. Shibata, A. H. Iwane, and T. Yanagida, Phys. Rev. Lett. 101, 128103 (2008).

[31] E. Homsher, F. Wang, and J. Sellers, Am. J. Physiol. 262, C714 (1992).

[32] D. Choquet, D. P. Felsenfeld, and M. P. Sheetz, Cell 88, 39 (1997).

[33] N. Gavara, R. Sunyer, P. Roca-Cusachs, R. Farré, M. Rotger, and D. Navajas, J. Appl. Physiol. 101, 512 (2006). 